\title{
T cell repertoire remodeling following post-transplant T cell therapy coincides with clinical response
}

\author{
Corey Smith, ${ }^{1,2}$ Dillon Corvino, ${ }^{1}$ Leone Beagley, ${ }^{1}$ Sweera Rehan, ${ }^{1}$ Michelle A. Neller, ${ }^{1}$ Pauline Crooks, ${ }^{1}$ Katherine K. Matthews, ${ }^{1}$ \\ Matthew Solomon, ${ }^{1}$ Laetitia Le Texier, ${ }^{1}$ Scott Campbell, ${ }^{3,4}$ Ross S. Francis, ${ }^{3,4}$ Daniel Chambers, ${ }^{4,5}$ and Rajiv Khanna ${ }^{1,4}$ \\ 'QIMR Centre for Immunotherapy and Vaccine Development, QIMR Berghofer Medical Research Institute, Brisbane, Queensland, Australia. 2School of Biomedical Sciences, Faculty of Health, Queensland \\ University of Technology, Brisbane, Queensland, Australia. ${ }^{3}$ Department of Nephrology, Princess Alexandra Hospital, Brisbane, Queensland, Australia. ${ }^{4}$ School of Clinical Medicine, The University of \\ Queensland, Brisbane, Queensland, Australia. ${ }^{5}$ Queensland Lung Transplant Service, The Prince Charles Hospital, Brisbane, Queensland, Australia.
}

\begin{abstract}
BACKGROUND. Impaired T cell immunity in transplant recipients is associated with infection-related morbidity and mortality. We recently reported the successful use of adoptive T cell therapy (ACT) against drug-resistant/recurrent cytomegalovirus in solid-organ transplant recipients.
\end{abstract}

METHODS. In the present study, we used high-throughput T cell receptor V $\beta$ sequencing and T cell functional profiling to delineate the impact of ACT on T cell repertoire remodeling in the context of pretherapy immunity and ACT products.

RESULTS. These analyses indicated that a clinical response was coincident with significant changes in the $T$ cell receptor V $\beta$ landscape after therapy. This restructuring was associated with the emergence of effector memory $T$ cells in responding patients, while nonresponders displayed dramatic pretherapy T cell expansions with minimal change following ACT. Furthermore, immune reconstitution included both adoptively transferred clonotypes and endogenous clonotypes not detected in the ACT products.

CONCLUSION. These observations demonstrate that immune control following ACT requires significant repertoire remodeling, which may be impaired in nonresponders because of the preexisting immune environment. Immunological interventions that can modulate this environment may improve clinical outcomes.

TRIAL REGISTRATION. Australian New Zealand Clinical Trial Registry, ACTRN12613000981729.

FUNDING. This study was supported by funding from the National Health and Medical Research Council, Australia (APP1132519 and APP1062074).

\section{Introduction}

In contrast to acute viral infections that are cleared from the body within a few weeks, human cytomegalovirus (CMV) establishes lifelong latency within the host following acute infection (1). CMV is ubiquitous worldwide, infecting most of the population in early childhood and adolescence (2). In the vast majority of individuals, infection is unrecognized and constant viral reactivation is controlled by $\mathrm{CD} 4^{+}$and $\mathrm{CD} 8^{+} \mathrm{T}$ lymphocytes, which are maintained at high frequencies in the periphery and patrol sites of latency $(3,4)$. These memory $\mathrm{T}$ cell populations display unique

Conflict of interest: CS and RK receive research and consultancy funding from Atara Biotherapeutics Inc. RK is also appointed as an advisor on the Atara Biotherapeutics Scientific Advisory Board. CS and RK hold international patents (US patent 2002312654) or patent applications (US provisional 62/340223) that cover CMV epitope sequences and their use in adoptive immunotherapy. RF has received nonfinancial support from Amgen Pharmaceuticals and Novartis Pharmaceuticals. SC has received non-financial support from Astellas, Merck Sharp \& Dohme, Shire, and Novartis, as well as personal fees from Novartis.

Copyright: () 2019, American Society for Clinical Investigation.

Submitted: February 21, 2019; Accepted: August 8, 2019; Published: October 14, 2019

Reference information: J Clin Invest. 2019;129(11):5020-5032.

https://doi.org/10.1172/JCl128323. functional characteristics, including immediate effector function upon recognition of cognate antigen $(5,6)$. The $\mathrm{T}$ cell landscape in individuals asymptomatically infected with $\mathrm{CMV}$ is dominated by the stable oligoclonal expansion of antigen-specific T cells $(3,7)$. CMV-specific $\mathrm{T}$ cells therefore function to maintain equilibrium with reactivating virus for the entire human lifespan in most individuals. However, this equilibrium is disrupted in transplant recipients who, owing to an intensive immunosuppressive regimen, may be unable to develop normal immunological memory $(8,9)$. This can manifest as chronic viral disease, leading to end-organ disease and graft loss (9). There is now compelling evidence in a number of solid-organ transplant (SOT) settings, including lung, kidney, and heart, that CMV reactivation and disease is associated with the failure to establish stable immunological memory against CMV (8, 10-12).

Despite clear evidence of the importance of efficient immunological surveillance in the prevention of CMV disease in SOT patients, current prophylactic and therapeutic interventions for CMV are dependent on the administration of antiviral therapy, rather than immunological reconstitution (13). While this is an efficient means to prevent viral reactivation, the emergence of drug resistance or intolerance results in viral reactivation that is 
Table 1. Clinical characteristics of patients included in the current study

\begin{tabular}{|c|c|c|c|c|c|c|c|c|c|}
\hline & $\begin{array}{l}\text { Patient } \\
\text { code }\end{array}$ & Organ & $\begin{array}{l}\text { CMV clinical indications } \\
\text { (peak viremia) }\end{array}$ & $\begin{array}{l}\text { Donor/recipient } \\
\text { CMV status }\end{array}$ & Immunosuppression & $\begin{array}{l}\text { Antiviral } \\
\text { treatment }\end{array}$ & $\begin{array}{l}\text { T cell dose } \\
\left(\times 10^{6}\right)\end{array}$ & $\begin{array}{l}\text { CMV specificity (\%) } \\
\text { of CD8 }{ }^{+} \text {cells }\end{array}$ & $\begin{array}{l}\text { Response to } \\
\text { therapy }\end{array}$ \\
\hline \multirow[t]{5}{*}{ Responders } & PCHO1 & Lung & $\begin{array}{l}\text { Esophagitis; viremia } \\
\qquad\left(8 \times 10^{3}\right)\end{array}$ & $-1-$ & EVR, PRD & GCV, FOS & 210 & 56.9 & $\begin{array}{c}\text { CR; cessation of antiviral } \\
\text { therapy }\end{array}$ \\
\hline & $\mathrm{PCHO4}$ & Lung & $\begin{array}{l}\text { Pneumonitis, colitis; } \\
\text { recurrent viremia } \\
\qquad\left(17 \times 10^{3}\right)\end{array}$ & $+/-$ & $\begin{array}{l}\text { CSA, TAC, MMF, } \\
\quad \text { EVR }\end{array}$ & $\begin{array}{l}\text { VGCV, GCV, } \\
\text { FOS, IVIG, LEF }\end{array}$ & 168 & 63.6 & $\mathrm{PR}$; reduction in viremia \\
\hline & PCHO6 & Heart & $\begin{array}{l}\text { Contraindicated to } \\
\text { antiviral therapy }\end{array}$ & $+/+$ & CSA, MMF & VGCV & 204 & 71.4 & $\begin{array}{c}\text { CR; cessation of antiviral } \\
\text { therapy }\end{array}$ \\
\hline & РAH06 & Kidney & $\begin{array}{l}\text { Colitis; recurrent viremia } \\
\qquad\left(12 \times 10^{3}\right)\end{array}$ & $-1-$ & TAC, MMF, PRD & VGCV, GCV & 245 & 77.2 & $\begin{array}{l}\text { CR; reduction in viremia; CMV } \\
\text { disease symptoms resolved }\end{array}$ \\
\hline & РAHO9 & Kidney & $\begin{array}{l}\text { Recurrent viremia } \\
\left(10 \times 10^{3}\right) \text {; diarrhea }\end{array}$ & $+/-$ & $\begin{array}{c}\text { TAC, MMF, PRD, } \\
\text { MePRD }\end{array}$ & VGCV, GCV & 180 & 48.4 & $\begin{array}{l}\text { CR; diarrhea resolved; } \\
\text { resolution of viremia }\end{array}$ \\
\hline \multirow[t]{2}{*}{ Nonresponders } & PCHO3 & Lung & $\begin{array}{l}\text { Pneumonitis; recurrent } \\
\text { viremia }\left(12 \times 10^{3}\right)\end{array}$ & $+/-$ & $\begin{array}{l}\text { TAC, MMF, EVR, } \\
\text { AZA, MMF }\end{array}$ & $\begin{array}{l}\text { VGCV, GCV, } \\
\text { FOS }\end{array}$ & 42 & 63.6 & $\begin{array}{l}\text { NR; died of multi-organ } \\
\text { failure }\end{array}$ \\
\hline & RAH01 & Lung & $\begin{array}{l}\text { Pneumonitis; recurrent } \\
\text { viremia }\left(19 \times 10^{3}\right)\end{array}$ & $+/-$ & TAC, PRD & VGCV, GCV & 104 & 31.9 & $\begin{array}{l}\text { NR; ongoing elevated } \\
\text { viremia }\end{array}$ \\
\hline
\end{tabular}

AZA, azathioprine; CR, complete response; CSA, cyclosporin A; EVR, everolimus; FOS, foscarnet; GCV, ganciclovir; IVIG, intravenous immunoglobulin; LEF, leflunomide; MePRD, methylprednisolone; MMF, mycophenolate mofetil; NR, no response; PR, partial response; PRD, prednisolone; TAC, tacrolimus; VGCV, valganciclovir.

often treated with secondary antiviral therapy such as foscarnet, which can lead to significant adverse effects that most patients do not tolerate long-term $(13,14)$. CMV-specific immune reconstitution offers an alternative approach to prevent ongoing viral complications without the need for long-term antiviral therapy. We have developed an adoptive T cell therapy (ACT) approach to restore immunological control in patients with CMV complications. This approach is based on a 2 -week in vitro expansion of autologous CMV-specific T cells using a customized peptide pool containing 30 HLA class I and II-restricted CMV-encoded epitopes. Following 2 case reports of successful treatment of lung transplant recipients with autologous CMV-specific ACT $(15,16)$, we recently completed an open-label phase I clinical study treating SOT recipients (lung, kidney, and heart) with autologous CMV-specific ACT (17). In total, we have now treated 13 patients with autologous CMV-specific ACT and met our primary objective of demonstrating safety, with no evidence of severe adverse events or graft-related events associated with $\mathrm{T}$ cell infusion. In our clinical studies, 11 of 13 patients showed evidence of a clinical response to therapy, predominantly characterized by a reduction in viral load in the peripheral blood or an improvement in organ-specific disease symptoms. The remaining 2 patients showed no benefit from ACT. In this follow-up study, we sought to explore the impact ACT has on the peripheral $\mathrm{T}$ cell repertoire and explore potential correlates of improved clinical outcome in responding patients.

\section{Results}

Clinical responses to CMV-specific ACT are associated with changes in the $T$ cell receptor $V \beta$ repertoire. A summary of clinical data from the SOT recipients included in the current study is provided in Table 1, and a schematic representation of the current study strategy is provided in Figure 1. Ten participants from our previously published clinical trial (17) were selected for the current analysis based on the availability of peripheral blood mononuclear cells (PBMCs) following ACT. These patients received CMV-specific T cells (median, $1.92 \times 10^{8}$; range, $4.2 \times 10^{7}$ to $2.45 \times 10^{8}$ ) in 2-6 intravenous infusions (Table 1 ). CMV-specific T cells expanded from all SOT recipients showed strong virus-specific reactivity (median, 57.1\%; range, $22.9 \%-77.2 \%$ ), and the majority of cell therapy products were dominated by $\mathrm{CD}^{+} \mathrm{T}$ cells (Table 1 ), with no detectable virus-specific $\mathrm{CD} 4^{+} \mathrm{T}$ cells. Eight of the ten patients, characterized as responders, displayed evidence of improvement in CMV clinical symptoms, demonstrated by a reduction in viremia, end-organ disease resolution, and/or cessation of antiviral therapy following ACT (Table 1). Of the 2 nonresponding patients, one died of multi-organ failure with no improvements in clinical symptoms, and the other displayed ongoing elevated viremia. We first aimed to delineate the impact of ACT on the SOT recipients' global $\mathrm{CD}^{+} \mathrm{T}$ cell repertoire, functional $\mathrm{CD} 8^{+}$ $\mathrm{T}$ cell landscape, and clinical response. $\mathrm{CD} 8^{+} \mathrm{T}$ cells were sorted from peripheral blood samples collected either before the commencement of $\mathrm{T}$ cell therapy (pretherapy), 4-8 weeks after 


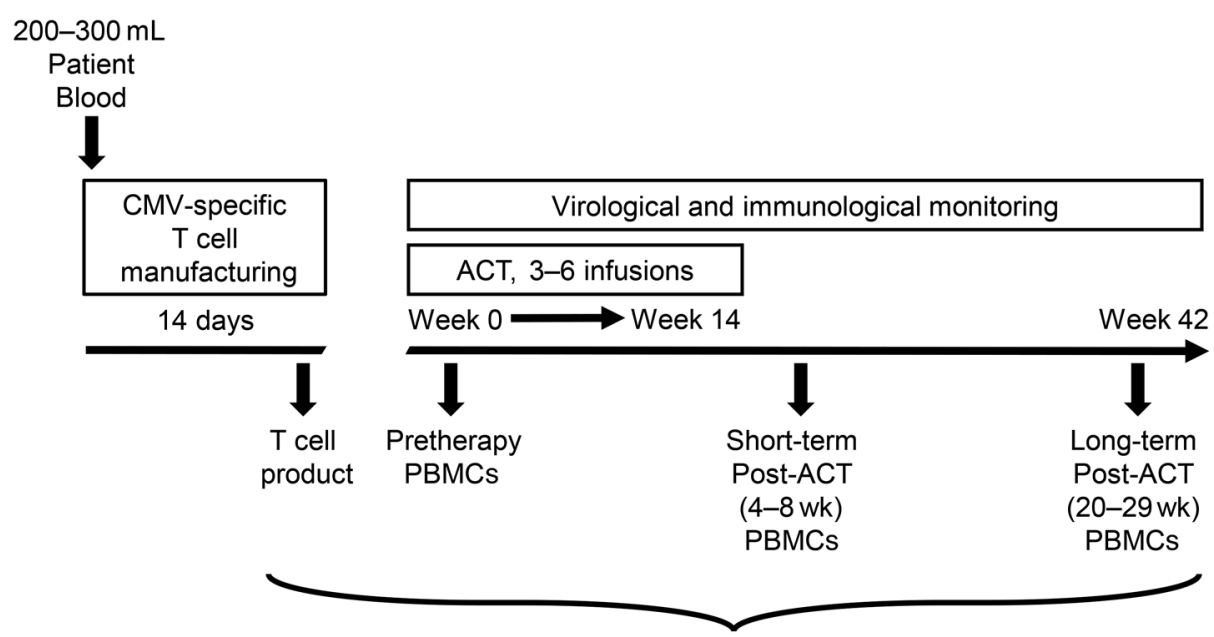

Functional, phenotypic, and TCR repertoire analysis
Figure 1. Schematic representation of the study. Autologous CMV-specific T cells were expanded in vitro for 2 weeks. Patients received up to 6 doses of autologous CMV-specific T cells over a period of 14 weeks. Patients were monitored for up to 29 weeks following the final infusion. PBMC samples preinfusion, short-term post-ACT (4-8 weeks), and long-term post-ACT (20-29 weeks) were used to assess $\mathrm{CD} 8^{+} \mathrm{T}$ cell characteristics. the completion of therapy (post, short term [post ST]), or 20-29 weeks after the completion of therapy (post, long term [post LT]), and $\mathrm{T}$ cell receptor $\mathrm{V} \beta$ (TRBV) deep sequencing analysis was performed. A summary of the TRBV analysis is provided in Supplemental Table 1 (supplemental material available online with this article; https://doi.org/10.1172/JCI128323DS1). Before the commencement of therapy, patients displayed disparate TRBV clonotypic compositions. Despite ongoing complications with CMV, some patients displayed dramatic oligoclonal expansions associated with CMV exposure, whereas others showed very little repertoire bias (Figure 2A). The TRBV complementarity-determining region 3 (CDR3) landscape in these patients, exemplified by patient PAHO8, was dominated by low-frequency clonotypes. This lack of pretherapy clonal expansion was associated with a more dramatic change in the TRBV landscape post-ACT. Patients PAH08, PAHO9, PCHO2, and PCHO4 displayed the greatest restructuring of their CD8 TRBV repertoire following ACT, characterized by a reduction in diversity and a dramatic increase in clonality (Figure 2B). The fold change in clonality after therapy inverse-

Figure 2. CD8+ $\mathrm{T}$ cell clonality in SOT recipients following adoptive immunotherapy. (A) Data represent the proportion of productive rearrangements when clones are grouped by frequency into small, medium, large, or hyperexpanded. (B) Data represent the productive clonality in SOT recipients before and after immunotherapy. (C) Data show a correlation between fold change in productive clonality long-term post-therapy and T cell clonality prior to the commencement of ACT. Significance was determined using a 2-tailed nonparametric Spearman's correlation.
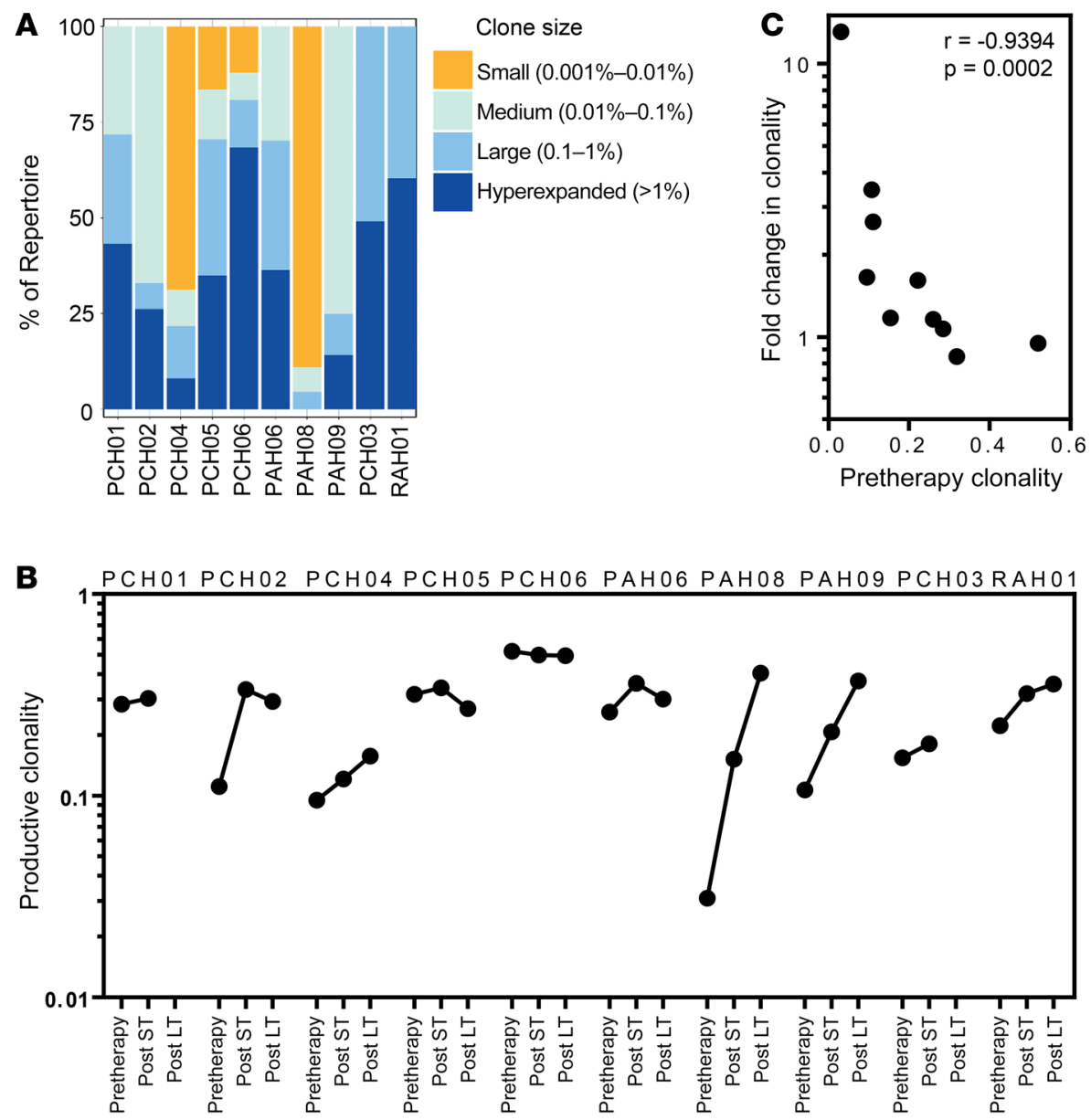

ly correlated with the pretherapy clonality (Figure 2C), suggesting that patients with a less biased repertoire were more receptive to ACT-mediated changes in the TRBV landscape.

Changes in clonotypic composition following ACT are associated with significant clonotypic expansion. To further delineate the impact of ACT on T cell repertoire, we used a 2-sided binomial test to determine the number of clonotypes in each patient that displayed significant changes following ACT. All SOT recipients 
Table 2. CMV-specific clonotypes identified in SOT recipients treated with ACT

\begin{tabular}{|c|c|c|c|c|c|}
\hline Epitope & Patient & TRBV CDR3 & Pretherapy & Post ST & Post LT \\
\hline \multirow[t]{4}{*}{ VTEHDTLLY } & PCHO2 & CASSLSGSYEQYF & 0 & 0 & 0.01 \\
\hline & & CASSPGAVYEQYF & 0 & 0 & 0.01 \\
\hline & РАНО8 & CASSVGQGVTYEQYF* & 0 & 0.05 & 0.07 \\
\hline & & CASSWTGPSYEQYF & 0.01 & 0.03 & 0.02 \\
\hline \multirow[t]{25}{*}{ NLVPMVATV } & PCHO4 & CASSQDAGSSYNEQFF & 0.17 & 0.23 & 0.19 \\
\hline & & CASSFGGYEQYF & 0 & 0 & 0.01 \\
\hline & & CASSFSETQYF & 0 & 0 & 0.01 \\
\hline & & CASSPNTCELFF & 0 & 0 & 0.01 \\
\hline & PCHO5 & CASSFSSLNTCELFF & 1.14 & 0.94 & 0.64 \\
\hline & & CASSLGVNTEAFF & 0.01 & 0.01 & 0.02 \\
\hline & & CASSLSASGYNEQFF & 0.01 & 0 & 0 \\
\hline & & CASSLVNEQFF & 0.01 & 0.02 & 0 \\
\hline & & CASSQARGEQYF & 0.01 & 0 & 0 \\
\hline & & CSARDGTGNGYTF & 0.01 & 0.01 & 0 \\
\hline & PCHO6 & CASSSANYGYTF & 35.04 & 22.45 & 19 \\
\hline & & CASSYATGTAYGYTF* & 0.59 & 1.3 & 1.99 \\
\hline & & CASSLAPCATNEKLFF* & 0.01 & 0.15 & 0.35 \\
\hline & & CASSSVTEAFF & 0.13 & 0.14 & 0.09 \\
\hline & & CASSLAPGTTNEKLFF & 0.02 & 0.03 & 0.04 \\
\hline & & CASSLVGGRYGYTF & 0.04 & 0.03 & 0.02 \\
\hline & & CASASANYCYTF & 0.01 & 0.02 & 0.02 \\
\hline & & CASSPGTCHTCELFF & 0 & 0.02 & 0.02 \\
\hline & & CASSLQACANEQFF & 0 & 0.01 & 0.01 \\
\hline & & CASSLGVNEQFF & 0 & 0 & 0.01 \\
\hline & & CASSLEGYTEAFF & 0.02 & 0.01 & 0 \\
\hline & & CASSSAYYGYTF & 0 & 0.01 & 0 \\
\hline & РАHO6 & CASSLGVNTEAFF & 0 & 0 & 0.01 \\
\hline & & CASSYFGGNTEAFF & 0 & 0.01 & 0 \\
\hline & & CASSYNTEAFF & 0.02 & 0 & 0 \\
\hline \multirow[t]{4}{*}{ TPRVTGGGAM } & PCHO5 & CASSYSGNTEAFF & 0 & 0 & 0.01 \\
\hline & РАHO6 & CASSSRDRGSYEQYF & 0.67 & 0.33 & 0.21 \\
\hline & & CASSLSPSTGNYGYTF & 0.57 & 0.6 & 0.32 \\
\hline & & CASSYSGNTEAFF & 0 & 0 & 0.01 \\
\hline RPHERNGFTVL & РАHO6 & CASSPSRNTEAFF & 0 & 0.02 & 0.01 \\
\hline \multirow[t]{2}{*}{ QIKVRVDMV } & РАНО8 & CASSRLAGADTQYF & 0 & 0 & 0.01 \\
\hline & & CASSRLAGGTDTQYF* & 0 & 0 & 0.03 \\
\hline TRATKMQVI & PCHO4 & CASSQEMQGAYGYTF & 0.19 & 0.28 & 0.28 \\
\hline
\end{tabular}

*Significantly expanded clonotypes.

whose clinical symptoms resolved following ACT showed significant clonotypic expansion post-ACT (Figure 3A and Supplemental Figure 1). However, only 1 expanded clonotype was detected from the nonresponding patient, RAH01 (Figure 3B). Similarly, patient $\mathrm{PCHO}$ showed no expanded clonotypes following ACT. While this observation may reflect the reduced sample coverage in these individuals due to poor $\mathrm{CD} 8^{+} \mathrm{T}$ cell yield from PBMCs, it does suggest an association between outcome and clonotypic expansion post-ACT. While the most dramatic changes were evident in PAH08, in whom we observed a significant increase in 334 different clonotypes post-therapy, other responders, particularly PCH06, who did not display a change in clonality post-therapy, showed clear evidence of significant clonal expansion. This included the emergence of clonotypes that were undetectable before
ACT, indicative of the emergence of novel clonotypes that likely played a role in immune control of CMV infection and/or disease following ACT. We also noted that a number of CMV-specific clonotypes were present in these expanded populations, in addition to other CMV-specific clonotypes that did not display significant expansion post-therapy (Table 2). These significantly expanded clonotypes constituted more than $15 \%$ of the productive TRBV repertoire in all responding patients long-term post-ACT (Figure 3C). Most patients displayed bias in their TRBV usage, with significant changes detected in multiple TRBV families, which demonstrates the diversity of changes post-ACT.

To examine the relationship between the expanded clonotypes, we next performed grouping of lymphocyte interactions by paratope hotspots (GLIPH) analysis to determine the immunodominant CDR3 motifs in SOT recipients following ACT (18). In brief, GLIPH analysis considers all possible amino acid sequences (K-mers) of length 4 in a population of CDR3s and reports K-mers that are statistically overrepresented (read motif) when compared with a naive repertoire database. In most responding patients, we were able to identify motifs within the CDR3 sequences that expanded post-ACT (Supplemental Table 2). Representative analyses displaying motifs of greater than $0.1 \%$ frequency either preor post-ACT in 3 responders are shown in Figure 3D. These analyses suggest that changes in the CDR3 landscape, associated with a dramatic increase in clonality and/or the significant expansion of virus-specific clonotypes, were associated with an improved outcome in treated patients.

ACT products from different patients display unique specificity and structural composition. We next explored the association between $\mathrm{T}$ cell clonotypes within each ACT product and changes in TRBV repertoire landscape following ACT. The ACT products generated for most patients contained multiple CMV specificities restricted through 1 or more HLA alleles (Figure 4A). CMV-specific T cells enriched from ACT products using a cytokine capture protocol (Supplemental Figure 2 and Supplemental Table 3) showed a range of diversities with predominantly unique clonotypic architecture. Circos plots from the individual patients demonstrate little overlap in the pairing of TRBV and TRBJ genes, indicating unique $\mathrm{T}$ cell receptor usages in the different ACT products (Figure 4B).

To further assess the relationships between the clonotypic compositions of ACT products from different patients, we compared TRBV and CDR3 usage. A small number of ACT products displayed overlap in TRBV usage (Figure 5A) and CDR3 sequences, and this was most evident in patients $\mathrm{PAHO} 9$ and $\mathrm{PCHO}$, who shared 23 clonotypes and had an immunodominant response to the HLA-C ${ }^{*} 06: 02$-restricted TRA epitope (Figure 5B). However, very little overlap in clonotypic composition was seen between most patients' ACT products, despite the presence of CMV-specific $T$ cells recognizing the same epitopes. We also saw no clear differences in TRBV usage and clonotypic composition when comparing the ACT products of responding and nonresponding patients.

Post-ACT immune reconstitution is associated with the clonotypes present in the ACT product. To examine the reconstitution of clonotypes associated with the ACT products, we identified the top 20 dominant clonotypes in each product. These are represented in Figure $6 \mathrm{~A}$ as a proportion of the total productive rearrangements 
A

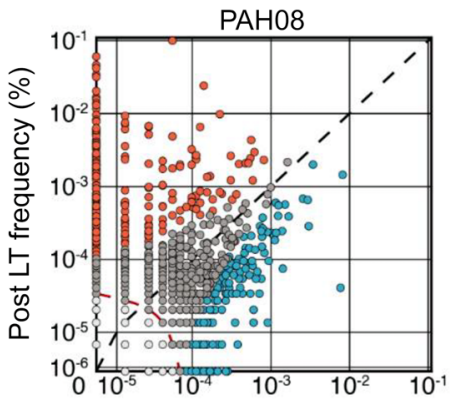

B

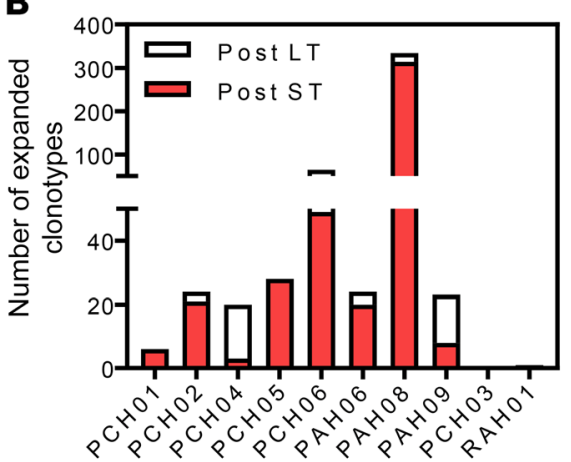

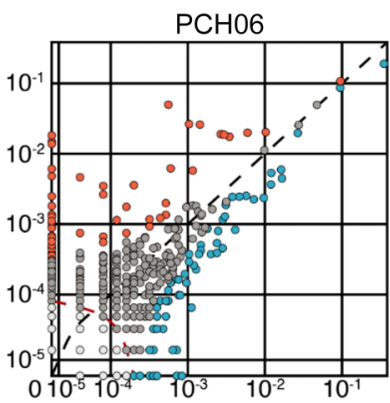

Pretherapy frequency (\%)
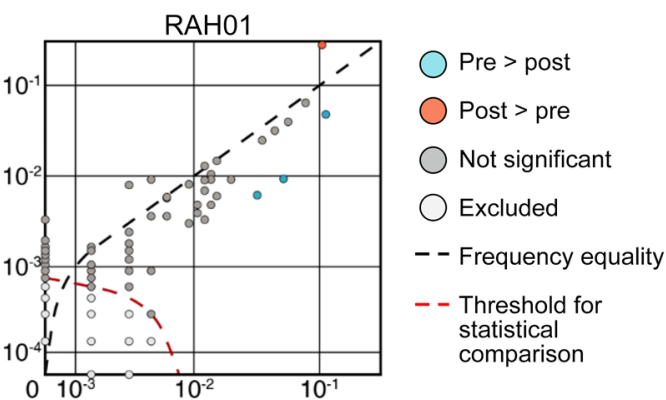

C

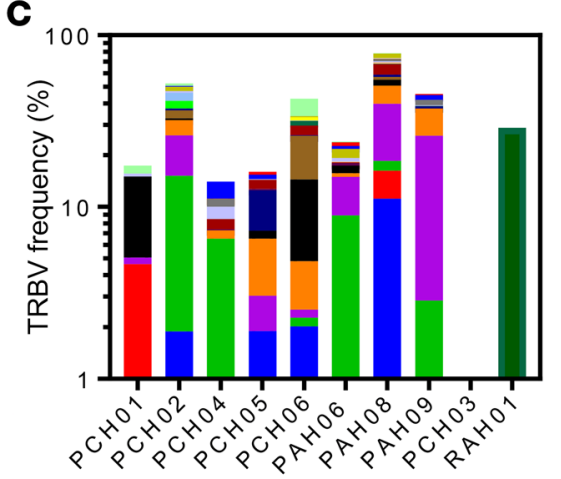

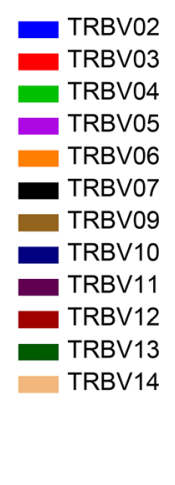

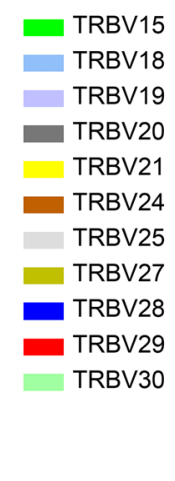

D

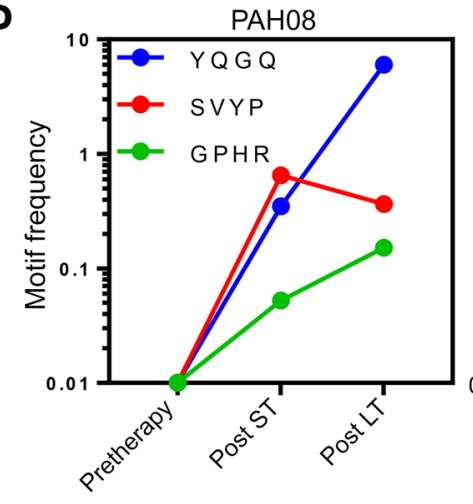

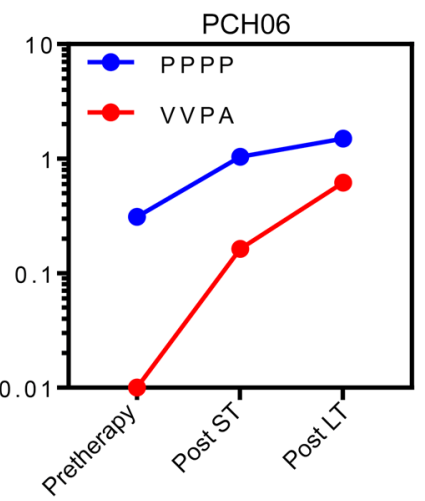

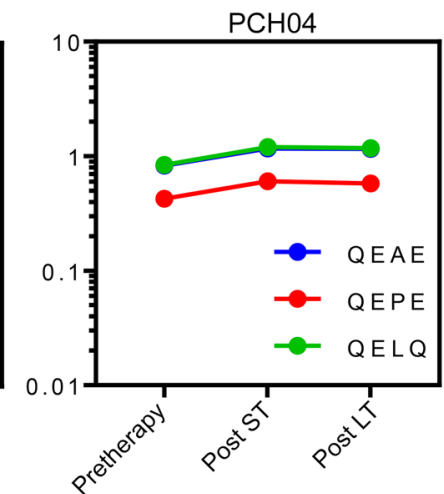

Figure 3. Changes in the clonotypic composition of the peripheral blood CD8 ${ }^{+} \mathbf{T}$ cell repertoire following adoptive immunotherapy. Significant changes in the frequency of patient $C D 8^{+} T$ cell clonotypes following adoptive cellular therapy were determined using the immunoSEQ platform. Significance was assessed in CDR3 sequences with a minimum of 5 reads. Significance was determined using a 2-sided binomial test with Benjamini-Hochberg multiple-comparisons correction, where $\alpha=0.01$. (A) Representative analyses from 3 patients comparing the frequency of T cell clonotypes in preinfusion and long-term post-therapy blood samples. Significantly expanded clonotypes post-therapy are shown in red. Clonotypes that show a significant reduction post-therapy are shown in blue. (B) Data represent the number of clonotypes in each patient that displayed significant expansion post-therapy. (C) The frequencies of TRBV families detected in significantly expanded clonotypes from each patient, represented as a proportion of the total CD8 ${ }^{+} T$ cell population. (D) GLIPH analysis was performed to determine the relationship between expanded clonotypes. Data represent CDR3 motifs enriched after immunotherapy in 3 responding patients.

in each product. To assess changes in the frequency of these clonotypes, we tracked them over time following ACT (Figure 6B). Global clonotypic analysis revealed that an increase in the frequency of cell therapy-associated clonotypes was most evident in patients with a more diverse repertoire pretherapy, particularly patients PAH08, PCHO2, and PCH04. However, changes in frequency did not necessarily correlate with the pretherapy clonotypic diversity, nor with immunodominance in the ACT product. For patient PAH08, 2 immunodominant clonotypes that were present in pre-ACT PBMCs and detected at a high frequency in their ACT product declined following treatment, in contrast to the majority of their other clonotypes. Similarly, patient PCH06's immuno- dominant HLA-A2-restricted NLV epitope-specific clonotype (see Table 1) declined post-therapy, while a significant expansion in other clonotypes specific for this epitope was seen.

Clonotypic expansion following ACT is not restricted to the clonotypes present in the ACT products. We next assessed the relationship between the clonotypic composition of the ACT product generated for each patient and the significantly expanded clonotypes detected post-therapy. We determined whether or not the significantly expanded clonotypes outlined in Figure 2 were detectable in the ACT product or PBMCs collected before the commencement of ACT, and what contribution these clonotypes made to the TRBV landscape post-therapy. Because of 
A

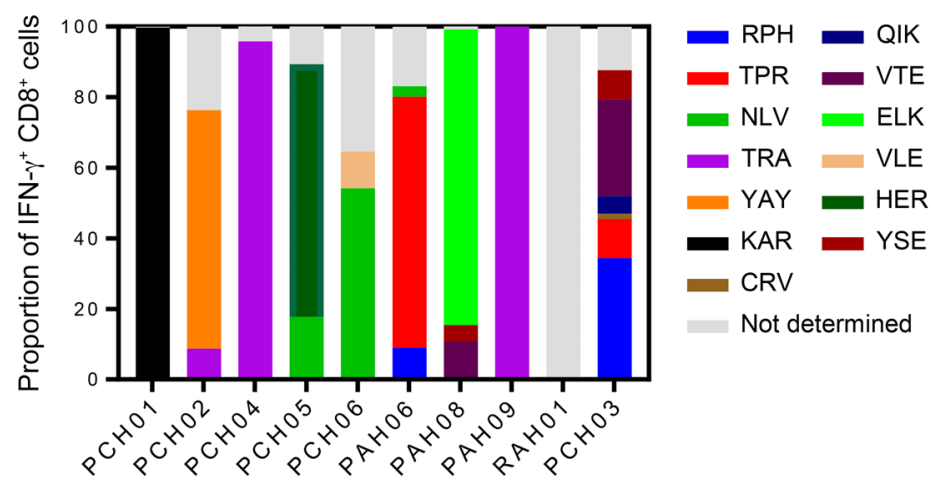

Figure 4. Characterization of the clonotypic composition of cell therapy products. (A) The frequency of $C D 8^{+} T$ cells recognizing CMV-encoded HLA-matched peptide epitopes was determined using a standard intracellular IFN- $\gamma$ assay. Data represent IFN- $\gamma$-producing $\mathrm{CD} 8^{+} \mathrm{T}$ cells responding to individual peptide epitopes as a proportion of the response detected with the CMV peptide pool containing all peptide epitopes. (B) Circos plots showing the $V$ and I gene pairings for $T$ cell receptor $\beta$ chain sequencing of therapy products. Ribbon thickness indicates number of pairings. Each color represents an individual TRBV or TRBJ family.
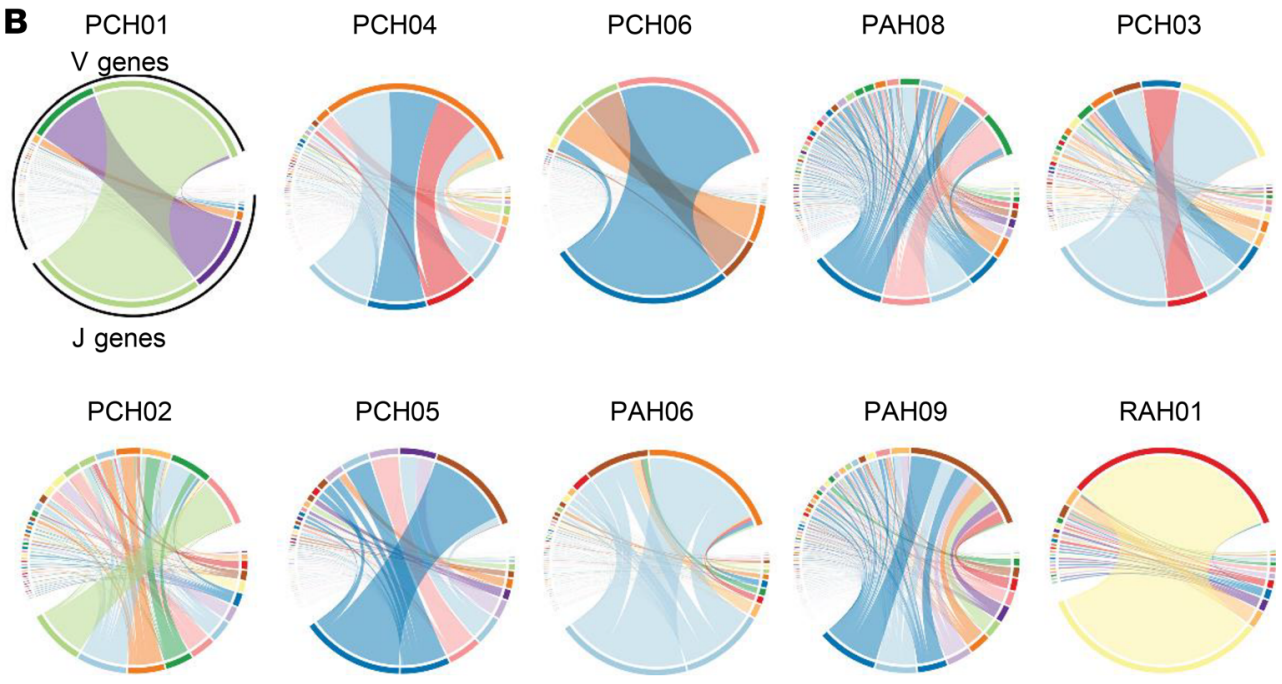

the low number of significant clonotypic expansions in the products of the 2 nonresponders ( 1 for patient RAH01 and none for patient PCH03), this analysis included only the 8 responders. The majority of SOT recipients who showed clinical improvement following ACT demonstrated a significant increase in the frequency of ACT product-associated clonotypes post-therapy (Figure 7). In these patients, we also observed the emergence of clonotypes that were undetectable pretherapy or in their ACT product. These observations indicate that immune reconstitution following CMV-specific ACT not only includes adoptively transferred clonotypes, but also impacts on antiviral immunity by promoting the expansion of endogenous clonotypes.

Immune reconstitution is associated with the expansion of effector memory $T$ cell populations post-therapy. To assess the relationship between changes in the CDR3 landscape and changes in CMV-specific $\mathrm{T}$ cell immunity, we analyzed the frequency of MHC multimer-specific T cells in the peripheral blood. This analysis was performed on 7 patients for whom MHC multimers were available. PBMCs from all SOT recipients contained CMV-specific $\mathrm{T}$ cells before immunotherapy, with the frequency of HLA-A2restricted NLV epitope-specific T cells exceeding 23\% in PCHO6, which correlated with the presence of the immunodominant NLV epitope-specific CDR3 sequence in the TRBV analysis (Figure 8A). While frequencies of CMV-specific T cells did not change dramatically post-ACT, the emergence of HLA-A ${ }^{*} 01: 01-$ restricted VTE epitope-specific T cells in participants $\mathrm{PCHO} 2$ and $\mathrm{PAHO}$, and
HLA-B ${ }^{*}$ 08:01-restricted ELK epitope-specific T cells in PAHO8, was observed. These changes were associated with an increase in the presence of cytokine-producing CMV-specific T cells. An increase in the frequency of HLA-C ${ }^{*} 06: 02-$ restricted TRA epitope-specific $\mathrm{T}$ cells was observed in the PBMCs of participant $\mathrm{PCHO} 4$, and was associated with an increase in the frequency of IFN- $\gamma$-producing antigen-specific T cells following ACT. While the HLA-A ${ }^{*}$ 2:01-restricted NLV epitope-specific T cell frequency in PCHO6 declined, this was not associated with a decrease in the frequency of functional T cells, suggesting that the new NLV epitope-specific clonotypes that emerged post-ACT constitute the functional component of this response.

To determine the phenotypic profile of the CMV-specific T cells pre- and post-ACT, we assessed the coexpression of CCR7, CD45RA, CD28, CD27, CD57, and CD95 in combination with MHC multimers. For each participant, samples were concatenated, and t-distributed stochastic neighbor embedding (tSNE) analysis was performed. This identified 6 predominant populations within global $\mathrm{CD}^{+} \mathrm{T}$ cells, characterized by differential surface marker expression (Supplemental Figure 3). The emerging CMV-specific $\mathrm{T}$ cell populations, represented by HLA-A ${ }^{*} 01: 01-$ restricted VTE epitope-specific T cells in patients PAHO8 and PCHO2, displayed an EM3 phenotype that was associated with high levels of CD57 expression and low expression of CD27 and CD28 (Figure 8B). This effector memory (EM) phenotype was also present post-ACT in the HLA-B ${ }^{*} 08: 01-$ restricted ELK epitope-specific T cells from 
A

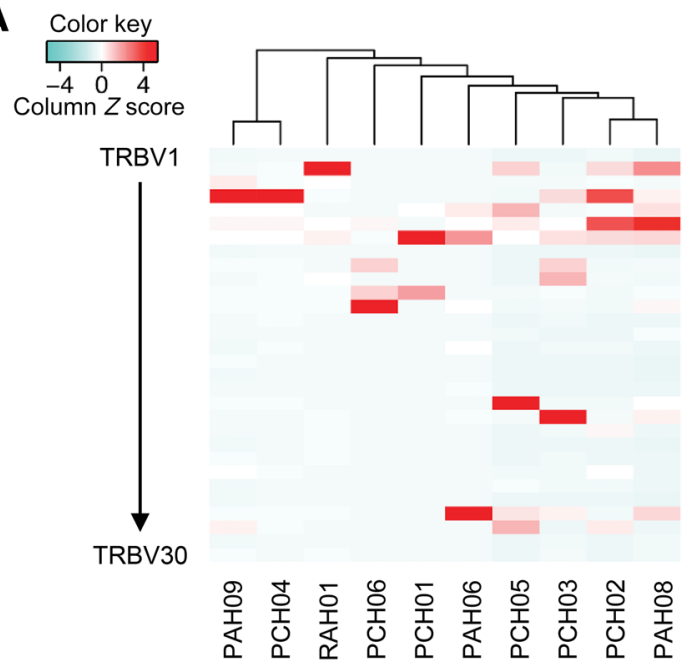

B
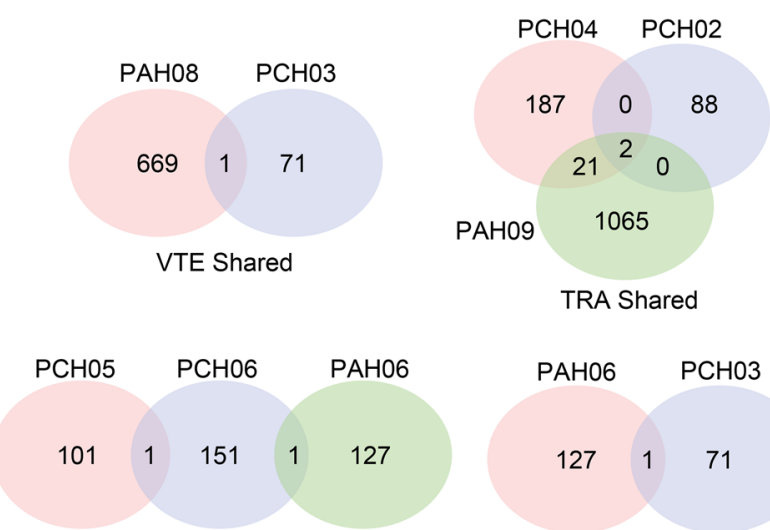

NLV Shared

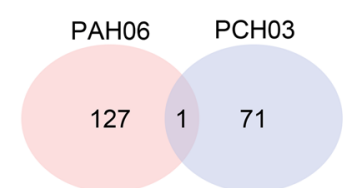

TPR Shared

Figure 5. TRBV and CDR3 clonotypic overlap between ACT products. (A) Heatmap showing the productive frequency of TRBV gene usage in cell therapy products. (B) The immunoSEQ platform was used to determine overlap in CDR3 sequences between patient cell therapy products that shared HLA-restricted peptide epitope responses. Venn diagrams represent the overlap between patients with an HLA-A*01:01-restricted VTE-specific response, an HLA-A*02:01-restricted NLV-specific response, an HLA-B*07:02-restricted TPR-specific response, and an HLA-C*06:02-restricted TRA-specific response.

PAH08 (data not shown). In contrast, the HLA-C*06:02-restricted TRA epitope-specific T cells from PAH09 and PCHO4 had a mixed phenotype, with both central memory (CM) and EM populations present before and after immunotherapy (Figure 8B). Similar observations of a mixed $\mathrm{CM} / \mathrm{EM}$ phenotype were also present in HLA-A ${ }^{*}$ 02:01-restricted NLV epitope-specific T cells in other patients (data not shown). These phenotypic differences are consistent with our previous observations in healthy virus carriers (19).

Immune reconstitution is associated with global $T$ cell phenotypic changes post-therapy. To more broadly explore the global T cell phenotype in trial participants before and after ACT, we used tSNE analysis to define memory populations in the global $\mathrm{CD}^{+} \mathrm{T}$ cell population. This analysis included 8 of the patients for whom post LT samples were available, excluding patients PCHO1 and $\mathrm{PCH}$ 03. To compare this analysis to functional $\mathrm{T}$ cell reconstitution in these patients, we assessed their CMV-specific polyfunctional cytokine profile through the expression of IFN- $\gamma$, TNF, and IL-2, and degranulation via CD107a surface expression. Polyfunctional cells were defined as those $\mathrm{T}$ cells capable of generating 2 or more functions (typically IFN- $\gamma^{+} \mathrm{CD} 107 \mathrm{a}^{+} \mathrm{TNF}^{+-}$). Monofunctional cells were those capable of producing only 1 function (typically IFN- $\gamma^{+}$). Supporting our observations from CDR3 analysis, $\mathrm{T}$ cells from the majority of the responding patients displayed proportional changes in memory profile following ACT, char-
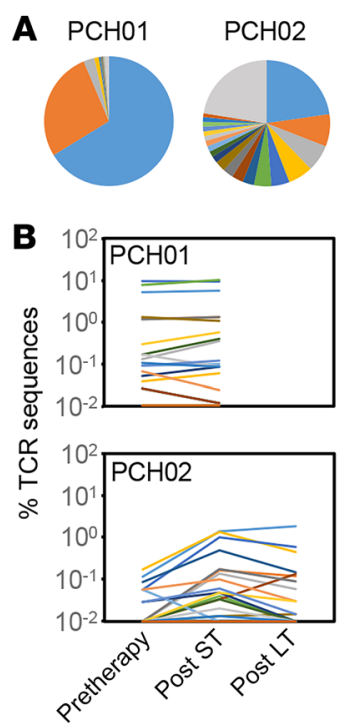
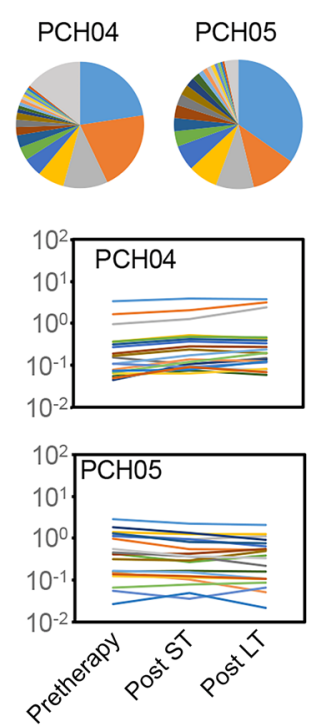
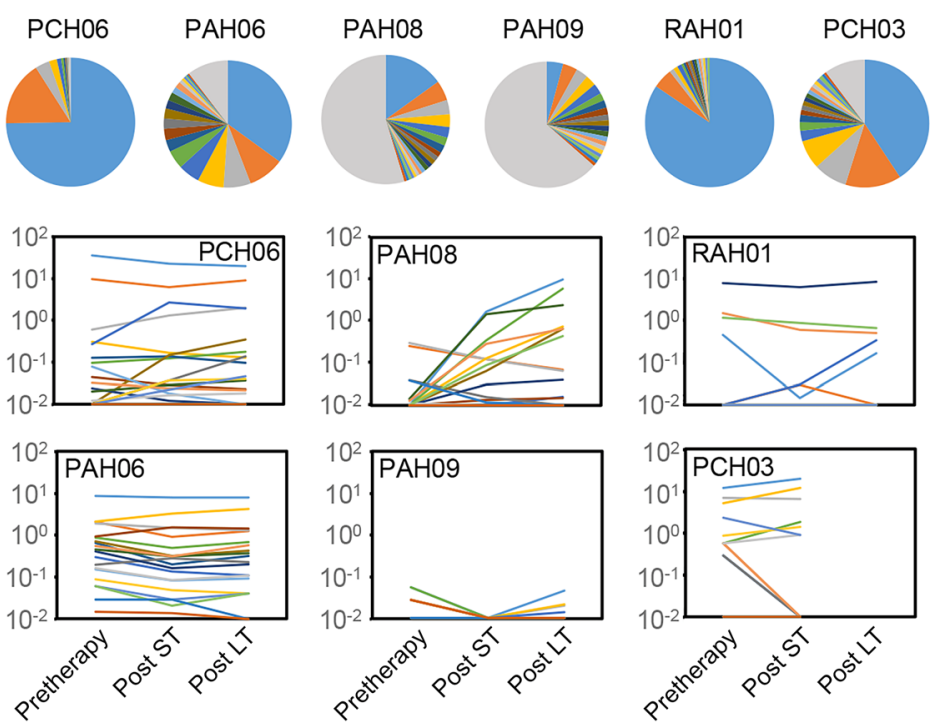

Figure 6. Reconstitution of cell therapy-associated clonotypes following adoptive immunotherapy. The 20 most prevalent CDR3 sequences in the cell therapy product from each patient were determined. (A) Colored slices in each pie chart represent the proportion of the top 20 clonotypes relative to productive clonotypes isolated from each cell therapy product. Gray slices represent CDR3 sequences that are not in the top 20 clonotypes. (B) The top 20 clonotypes from patient-specific cell therapy products were tracked over time in the corresponding patients. Colors match the CDR3 sequences in the pie charts in $\mathbf{A}$. 

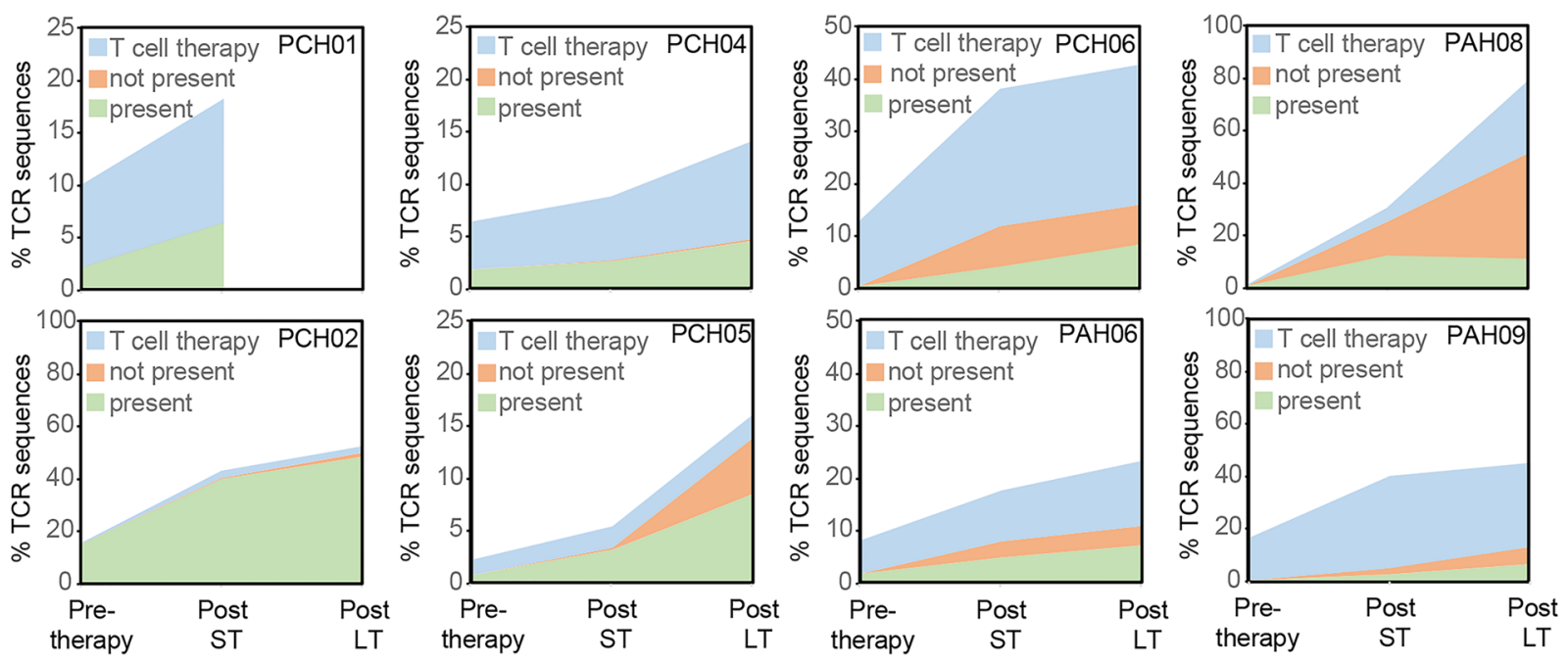

Figure 7. Tracking the association between significant clonotypic expansions and presence in the cell therapy products. The significantly expanded clonotypes from each patient (as outlined in Figure 1) were assessed for their presence in the T cell therapy products. Data represent the frequency of significantly expanded clonotypes associated with the T cell therapy (T cell therapy), present pretherapy but not detected in the cell therapy (Present), and not present pretherapy and not detected in the cell therapy (Not present).

acterized by an increase in the proportion of EM T cells (Figure 9A). The changes were particularly evident in $\mathrm{PCHO}$, $\mathrm{PCHO} 4$, $\mathrm{PAHO}$, and $\mathrm{PAH09}$, whose $\mathrm{CD}^{+} \mathrm{T}$ cell populations were dominated by naive T cells before ACT. These dramatic changes in clonotypic composition were associated with an expansion of EM3 $\mathrm{CD}^{+} \mathrm{T}$ cells in patients PCHO2, PCHO4, and PAHO8, and EM1, EM2, and EM3 T cells in PAH09. These patients also demonstrated an increase in the number of CMV-specific polyfunctional $\mathrm{T}$ cells following ACT (Figure 9B). Similarly, although a high proportion of EM3 cells was observed in $\mathrm{CD}^{+} \mathrm{T}$ cells from PCHO6 and PAHO6 prior to therapy, post-ACT samples also showed an increase in the proportion of EM cells, while a high frequency of EM T cells was maintained in PCHO5. These patients maintained a similar frequency of polyfunctional T cells following ACT. In contrast, participant RAH01 displayed a high proportion of both $\mathrm{CM}$ and EM populations pretherapy and negligible naive $\mathrm{T}$ cells (Figure 9). Consistent with our observation of very little change in the TRBV landscape in this patient and no evidence of virus-specific $\mathrm{T}$ cell reconstitution, this cellular phenotype did not change post-ACT. This patient also displayed very few polyfunctional $\mathrm{CD}^{+} \mathrm{T}$ cells following ACT.

Finally, to assess the potential impact the inflammatory milieu could be having on $\mathrm{T}$ cell expansion following ACT, we used a human cytokine/chemokine 65-plex panel to assess expression in plasma from the 10 ACT recipients before infusion. While we saw no clear patterns of differential cytokine or chemokine expression in responding patients that could reflect improved reconstitution following ACT, we noted elevated levels of G-CSF, IL-10, and IL-28A in plasma from one of our nonresponders, RAH01, prior to infusion (Supplemental Figure 4). In both nonresponders we also saw reduced expression of CCL15, a chemokine shown to promote recruitment into the lung. While these observations provide some indications of the potential impact the inflammatory milieu has on $\mathrm{T}$ cell reconstitution following ACT, they need to be further explored in a larger cohort or in a controlled preclinical model.

\section{Discussion}

In this study, we sought to explore the impact of adoptive antiviral $\mathrm{T}$ cell immunotherapy on the peripheral $\mathrm{T}$ cell landscape in SOT recipients who had developed $\mathrm{CMV}$-associated complications due to drug resistance or disseminated end-organ disease. Surprisingly, we noted significant diversity in the composition of the $\mathrm{CD} 8^{+} \mathrm{T}$ cell repertoire in responding patients prior to ACT; some patients displayed typical clonal expansions associated with persistent CMV infection, while others showed little bias in their repertoire. In the majority of trial participants who responded to ACT, changes in peripheral T cell repertoire were associated with dramatic restructuring of the $\mathrm{T}$ cell landscape and/or the expansion of virus-specific clonotypes following ACT. In contrast, a biased peripheral $\mathrm{T}$ cell repertoire that was not altered by ACT in nonresponding recipients suggests that the restructuring of the TRBV landscape following ACT was critical for the clinical improvements seen in the responding patients.

Persistent CMV infection has a dramatic impact on the $\mathrm{T}$ cell compartment in humans. This impact was initially demonstrated through the identification in seropositive individuals of expanded EM T cell populations (20), high frequencies of CMV-specific $\mathrm{T}$ cells, and the associated oligoclonal TRBV phenotype (3). More recent observations have shown the dramatic impact of CMV on global CD8 ${ }^{+}$TRBV architecture, characterized by a CMV-specific signature that can be used to differentiate exposed and nonexposed individuals (21). CMV reactivation and viral control in hematopoietic transplant recipients were recently associated with a rapid dramatic restructuring of both the EM and naive T cell repertoire (22). In contrast, we have previously reported that oligoclonal expansion can also be associated with poor viral control (23), indicative of the importance of $\mathrm{T}$ cell programming and functionality in promoting viral control (24). In the SOT setting, the establishment and maintenance of functional CMV-specific immunity have long been established via the analysis of cytokine production $(9,25,26)$, which has now been standardized for use in a number of diagnostic assays (27-29). Other recent observations have also demonstrated 
A
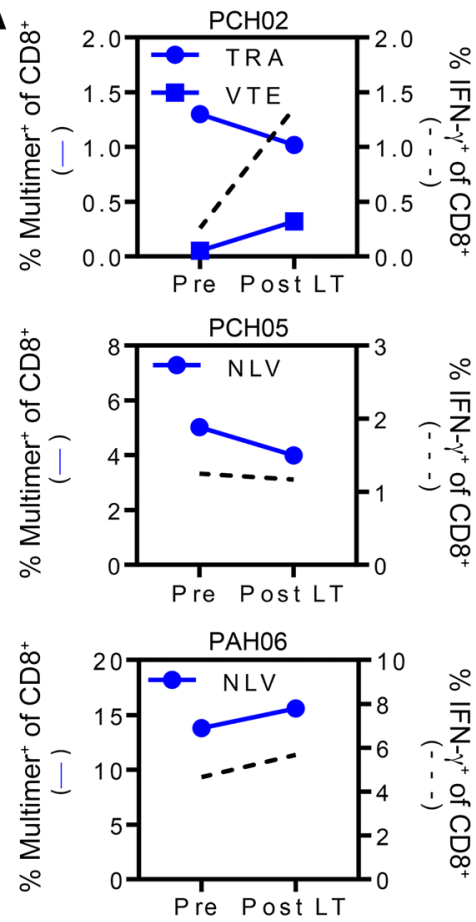

PAH09

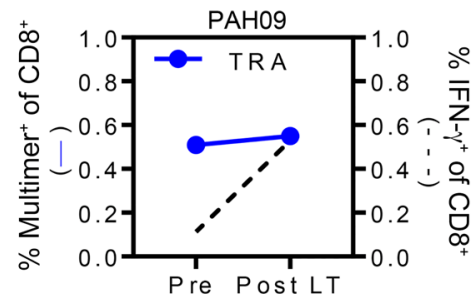

B
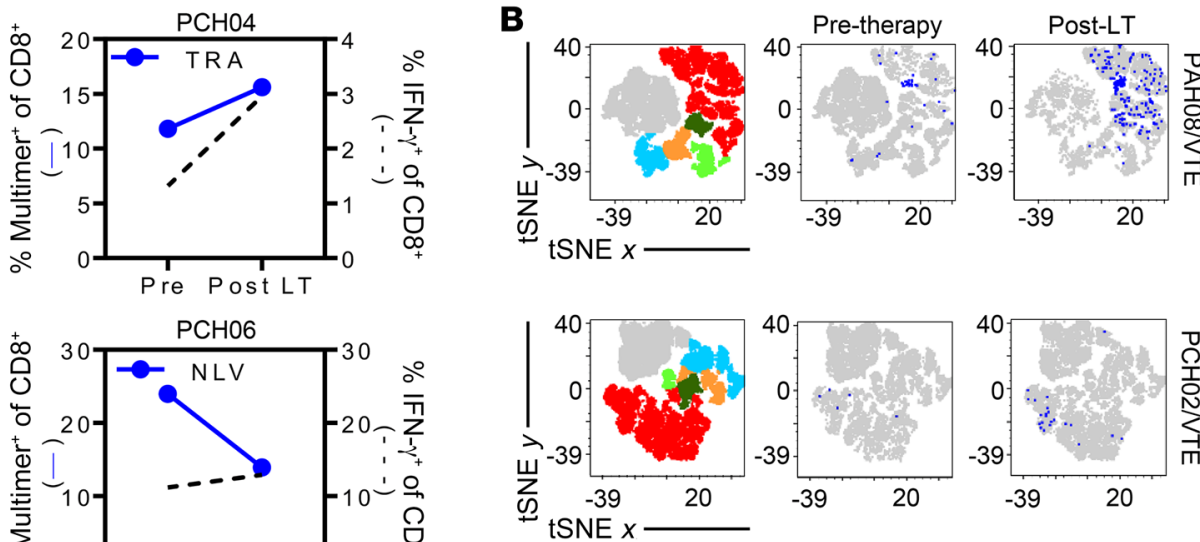
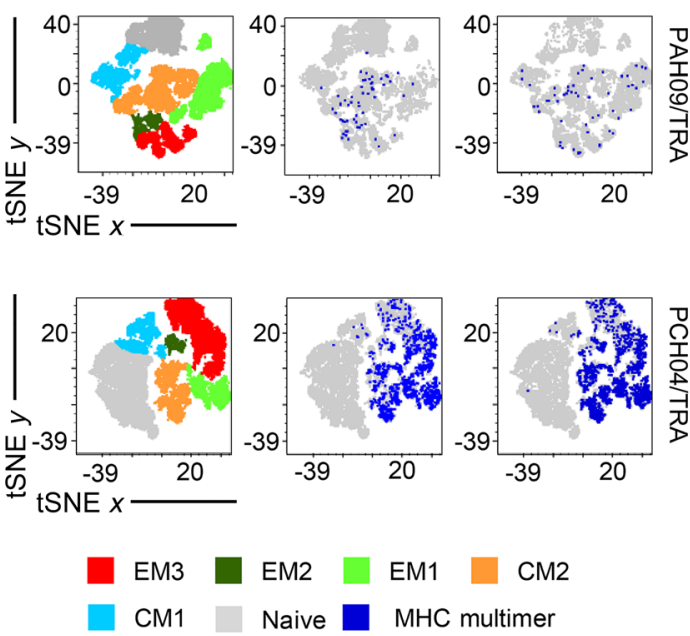

M1

Figure 8. CMV-specific T cell frequency and phenotype following ACT. The frequency and phenotype of CMV-specific T cells pretherapy and at long-term follow-up were assessed using HLA-matched MHC multimer analysis and the expression of CD27, CD28, CD45RA, CD57, CCR7, and CD95. (A) Data represent the frequency of HLA-matched MHC multimer-specific T cells (left axis) overlaid with the frequency of CMV-specific IFN- $\gamma$-producing CD8 ${ }^{+}$T cells (right axis). (B) Concatenated files were prepared from patient samples at each time point and tSNE analysis used to establish phenotypic populations in CD8 ${ }^{+}$ T cells, including naive, central memory (CM1 and CM2), and effector memory (EM1, EM2, and EM3). The gating strategy for each population and fluorescence intensity for each surface marker are shown in Supplemental Figure 3. Left panels represent the CD8 ${ }^{+} \mathrm{T}$ cell populations in 4 responding patients. Data in the middle and right panels are overlaid with the corresponding MHC multimer-specific population.

that efficient immunity against $\mathrm{CMV}$ is not just dependent on the presence of a high frequency of CMV-specific T cells, but also on their correct functional programming $(30,31)$. In the current study, we observed evidence for dramatic repertoire skewing prior to ACT despite underlying defects in viral control, further emphasizing the importance of functionality in these populations. While patients with little repertoire skewing appeared most responsive to ACT, it was evident that dramatic changes in the clonotypic composition could also occur in patients with existing oligoclonal expansions. Interestingly, clonotypic changes were not necessarily associated with preexisting immunodominance hierarchies or immunodominance hierarchies in the ACT products. This suggests that other properties of the $\mathrm{T}$ cells, such as phenotype and antigenic target, likely influence post-therapy expansion.

While our preliminary observations demonstrate an association between the control of CMV disease-related symptoms and the restructuring of the TRBV landscape following ACT, the mechanisms that prevent efficient $\mathrm{T}$ cell-mediated control in nonrespon- sive patients remain to be elucidated. However, it was clear that both nonresponding patients had preexisting clonal expansions. Although speculative, based on only 2 patients, it is plausible that underlying immunological defects associated with these dramatic clonotypic expansions may limit the ability of adoptively transferred $\mathrm{T}$ cells to expand and control virally infected cells. The removal of these populations through lymphodepletion could provide one strategy to improve the reconstitution of transferred $\mathrm{T}$ cells. This type of approach is successful in other settings of ACT, particularly in cancer patients $(32,33)$; however, it would also have the potential to deplete effector $\mathrm{T}$ cell populations that play a role in viral control. Lymphodepletion prior to ACT in SOT recipients would therefore need to preferentially target these oligoclonal EM $\mathrm{T}$ cell populations. However, this type of approach requires intensive treatment and would require larger cohort studies to better define the impact of the pre-ACT immunological environment on post-ACT immune reconstitution. An alternate approach would be to administer ACT prior to the dramatic changes in the TRBV landscape that are evi- 
A
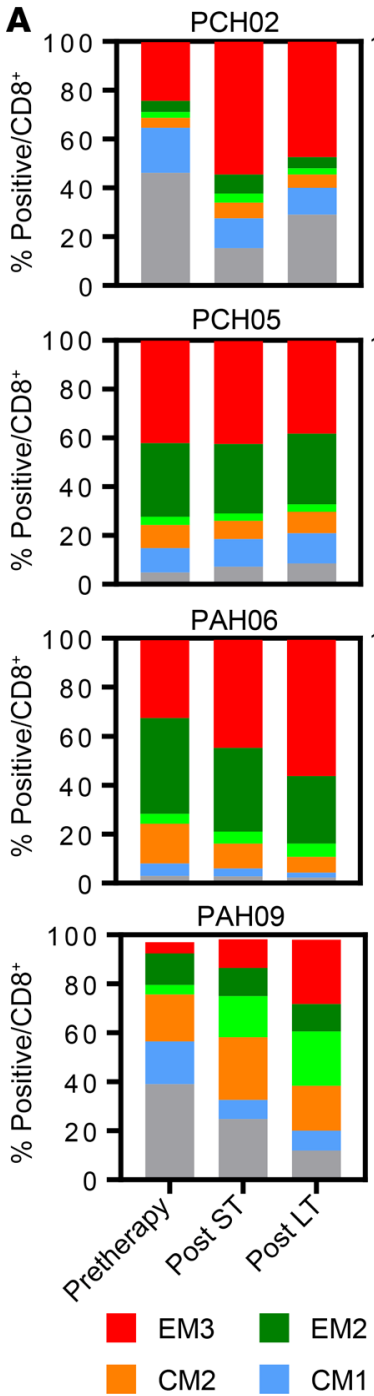

$\mathrm{PCHO} 4$

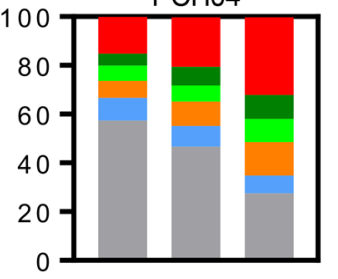

$\mathrm{PCHO6}$

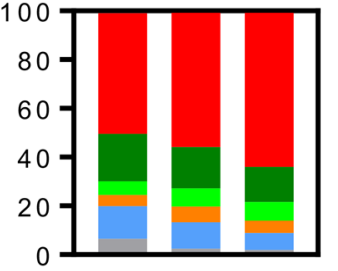

PAH08

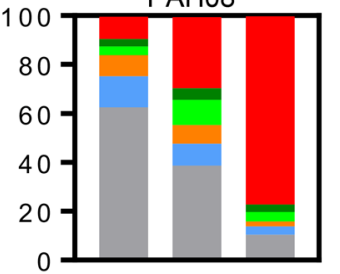

RAH01

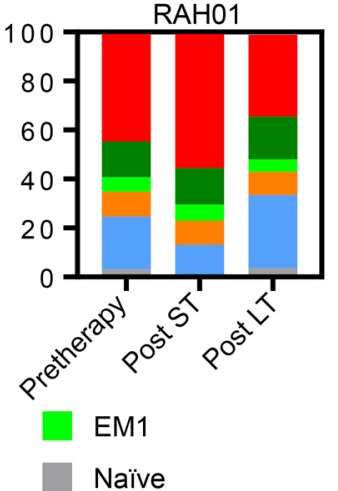

B
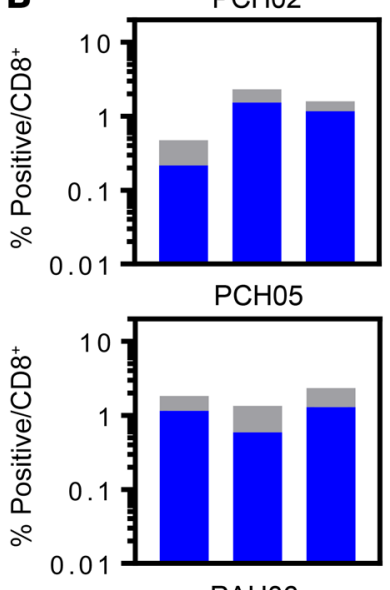

PAHO6
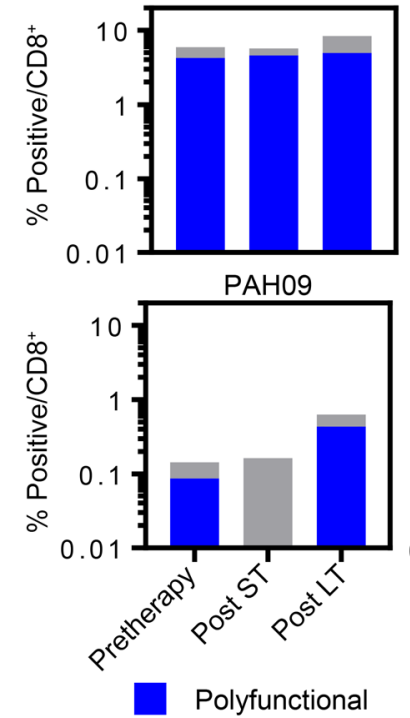

$\mathrm{PCHO4}$
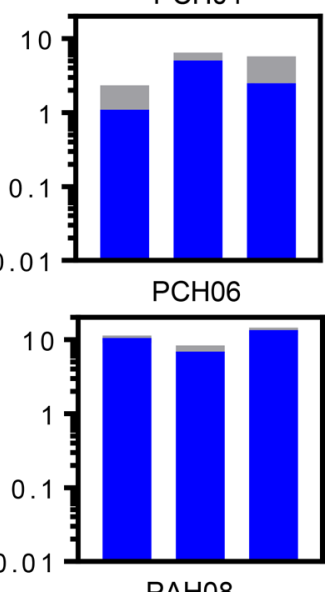

PAH08

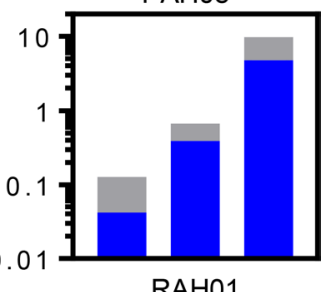

RAH01

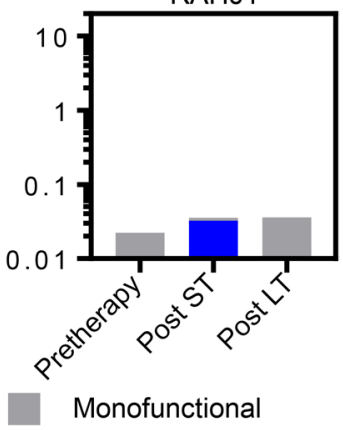

Figure 9. T cell phenotypic changes in SOT recipients following ACT. Concatenated files were prepared from patient samples at each time point and tSNE analysis used to establish phenotypic populations in CD8+ T cells. (A) Data represent the proportion of memory CD8+ $\mathrm{T}$ cell populations in total CD8+ $\mathrm{T}$ cells following ACT. (B) T cell polyfunctionality was assessed following stimulation with the CMV peptide pool used to generate the cell therapy. Polyfunctional cells were defined as those T cells exhibiting 2 or more functions (typically IFN- $\gamma^{+} \mathrm{CD} 107 \mathrm{a}^{+} \mathrm{TNF}^{+/-}$). Monofunctional cells were those capable of producing only 1 function (typically IFN- $\gamma^{+}$). Data represent the proportion of polyfunctional or monofunctional CD8 ${ }^{+} \mathrm{T}$ cells.

dent in some patients in our cohort. Although we cannot definitively say that immune control in patient PAHO8 was mediated by the administered $\mathrm{T}$ cells, this patient provides the strongest evidence that a lack of pretherapy clonal expansions is associated with post-therapy changes in the TRBV landscape. At-risk patients could be monitored for both viremia and CMV-specific immunity following viral reactivation, to allow intervention to occur during earlier stages of viral reactivation $(9,10)$. Other potential avenues to improve immune reconstitution following ACT could incorporate modulation of the cytokine environment prior to or during ACT. Although not definitive, our preliminary observations suggested some potential differences in the inflammatory milieu prior to cell therapy. Other confounding factors, including the ongoing coadministration of antirejection and antiviral medications, also likely have an impact on the efficacy of ACT. However, because of the limited size of the current cohort, it is not possible to assess this impact.
Another potential limitation on the success of autologous ACT for SOT patients is the quality of the cellular product. While we were unable to define clear differences in the ACT products of responders and nonresponders, it was evident that one of the nonresponders contained a near-monoclonal $\mathrm{T}$ cell population dominated by a single TRBV sequence. While this was also observed in some of the responding patients, $\mathrm{T}$ cell receptor diversity is known to play an important role in viral control $(34,35)$. Other unexplored functional defects are also likely present in ACT products generated from heavily immunosuppressed patients, which may impact the efficacy of these products. Another approach to treating viral complications, which has been well studied in hematopoietic stem cell transplant recipients, is the use of HLA-matched allogeneic virus-specific T cells $(36,37)$. This approach uses healthy donors as a source of PBMCs for the manufacture of T cells. It has the potential to provide cells selected to have optimal functionality, broad- 
er specificity, and increased clonotypic diversity from donors not exposed to heavily immunosuppressive drugs. It also allows for rapid administration of ACT from banked cryopreserved cells.

Cellular immunotherapy is emerging as a powerful strategy to restore immunological control against CMV and other common pathogens affecting SOT recipients and other immunosuppressed patients. Understanding the pretherapy immunological environment and post-therapy changes associated with immune control will provide a platform to better enhance the future clinical use of these approaches.

\section{Methods}

\section{Patient treatment}

The single-arm open-label phase I adoptive immunotherapy study, including the preparation of the ACT, has been previously described (17). Briefly, autologous CMV-specific T cells were generated from PBMCs using a custom-designed peptide pool that included 30 defined CMV-encoded epitopes. Patients received up to 6 doses of T cells every 2 weeks and were monitored for 29 weeks after the completion of therapy.

\section{Isolation and deep sequencing of $\mathrm{CD8}^{+} \mathrm{T}$ cells}

To isolate $\mathrm{CD} 8^{+} \mathrm{T}$ cells, $\mathrm{PBMCs}$ were incubated with anti-CD8-PerCPCy5.5 (clone RPA-T8, eBioscience), anti-CD4-FITC (clone RPA-T4, BD Biosciences), anti-CD3-APC (clone SK7, BD Biosciences), and LIVE/ DEAD Fixable Near-IR Dead Cell Stain (Thermo Fisher Scientific). $\mathrm{CD}^{+} \mathrm{CD}^{+} \mathrm{CD}^{-}$viable lymphocytes were sorted using a BD FACSAria III (Becton Dickinson). DNA was isolated from the sorted cells using Qiagen DNeasy Kit (Qiagen). To isolate IFN- $\gamma$-producing CD8 ${ }^{+} \mathrm{T}$ cells, T cells generated for ACT (ACT product) were stimulated with the CMV peptide pool and cultured for 4 hours. IFN- $\gamma$ was captured on cells using an IFN- $\gamma$ secretion assay (Miltenyi Biotec) with a PE-conjugated antiIFN- $\gamma$ detection antibody. Cells were costained with anti-CD8-PerCPCy5.5, anti-CD4-FITC, anti-CD3-APC, and LIVE/DEAD Fixable NearIR. $\mathrm{CD}^{+} \mathrm{CD}^{+} \mathrm{CD} 4-\mathrm{IFN}-\gamma^{+}$viable cells were sorted using a BD FACSAria III, and DNA isolated using the Qiagen DNeasy Kit. The isolated DNA was then sent to Adaptive Biotechnologies for TRBV deep sequencing analysis using the immunoSEQplatform.

\section{Analysis of TRBV sequencing}

Library preparation and sequencing. Sample data were generated using the immunoSEQ assay. The somatically rearranged human TRBV CDR3 was amplified from genomic DNA using a 2-step, amplification bias-controlled multiplex PCR approach $(38,39)$. Briefly, the first PCR consists of forward and reverse amplification primers specific for every $\mathrm{V}$ and J gene segment, and amplifies the hypervariable CDR3 of the immune receptor locus. The second PCR adds a proprietary barcode sequence and Illumina adapter sequences. CDR3 libraries were sequenced on an Illumina NextSeq 500 instrument according to the manufacturer's instructions.

Data analysis. Raw Illumina sequence reads were demultiplexed according to Adaptive Biotechnologies' proprietary barcode sequences. Demultiplexed reads were then further processed to remove adapter and primer sequences; identify and correct for technical errors introduced through PCR and sequencing; and remove primer dimer, germline, and other contaminant sequences. The data were filtered and clustered using both the relative frequency ratio between similar clones and a modified nearest-neighbor algorithm, to merge closely related sequences. The resulting sequences were sufficient to allow annotation of the $\mathrm{V}(\mathrm{N}) \mathrm{D}(\mathrm{N}) \mathrm{J}$ genes constituting each unique CDR3 and the translation of the encoded CDR3 amino acid sequence. V, D, and J gene definitions were based on annotation in accordance with the International Immunogenetics Information System (IMGT) database (www.imgt.org). The set of observed biological TRBV CDR3 sequences was normalized to correct for residual multiplex PCR amplification bias and quantified against a set of synthetic TRBV CDR3 sequence analogs (38). Initial alignment and bioinformatics were performed using the immunoSEQ Analyzer platform.

Additional analysis of TRBV was performed with VDJtools (version 1.1.10) (40) and the R package tcR (version 2.2.1.11) (41) using default settings, unless otherwise stated. GLIPH analysis was done using default settings in version 1.0 (18).

TCR sequencing data have been deposited in Adaptive Biotechnologies' immuneACCESS database (doi:10.21417/CS2019JCI; https://clients.adaptivebiotech.com/pub/smith-2019-JCI).

\section{Phenotypic characterization of PBMCs}

PBMCs were incubated with APC-labeled MHC class I multimers specific for the HLA-A ${ }^{*}$ 1:01-restricted epitope VTEHDTTLY (VTE), the HLA-A ${ }^{\star} 02: 01-$ restricted epitope NLVPMVATV (NLV), the HLA-B ${ }^{*}$ 07:02-restricted epitope TPRVTGGGAM (TPR) or RPHERNFGTVL (RPH), or the HLA-B ${ }^{\star} 08$ :01-restricted epitope ELKRKMIYM (ELK), supplied by Immudex; or the HLA-C ${ }^{\star} 06: 02-$ restricted epitope TRATKMQVI (TRA), supplied by the NIH Tetramer Core Facility (Emory University, Atlanta, Georgia, USA). Cells were then incubated with anti-CD4-PE-Cy7 (clone RPA-T4, BD Biosciences), anti-CD8-PerCPCy5.5 (clone RPA-T8, eBioscience), anti-CD19-PE-Cy5 (clone HIB19, BD Biosciences), anti-CD27-PE (clone L128, BD Biosciences), antiCD28-BV480 (clone CD28.2, BD Biosciences), anti-CD45RA-FITC (clone HI100, BD Biosciences), anti-CCR7-AF700 (clone 150503, BD Biosciences), anti-CD95-BV421 (clone DX2, BD Biosciences), antiCD57-BV605 (clone NK-1, BD Biosciences), and LIVE/DEAD Fixable Near-IR (Life Technologies). Cells were acquired using a BD LSRFortessa with FACSDiva software (BD Biosciences), and post-acquisition analysis was performed using FlowJo software (FlowJo LLC). To define phenotypically distinct $\mathrm{T}$ cell populations, $\mathrm{t}$-distributed stochastic neighbor embedding (tSNE) analysis was performed using FlowJo software. tSNE analysis was performed on each concatenated sample from each patient separately, and $\mathrm{T}$ cell populations were characterized based on surface marker expression (Supplemental Figure 3).

\section{Intracellular cytokine analysis of PBMCs}

To characterize the ACT products and PBMCs isolated from follow-up blood samples, cells were stimulated with CMV peptide epitopes and assessed for the expression of IFN- $\gamma$ (clone B27, BD Biosciences), TNF (clone Mab11, BD Biosciences), CD107a (clone H4A3, BD Biosciences), and IL-2 (clone MQ1-17H12, BD Biosciences) as previously described (17). Cells were acquired using a BD LSRFortessa with FACSDiva software. Post-acquisition analysis was performed using FlowJo software. See Supplemental Table 4 for a list of CMV epitopes used in this study.

\section{Analysis of plasma cytokine and chemokine profile}

To explore the inflammatory milieu in patients before the commencement of ACT, plasma was harvested from peripheral blood and stored at $-70^{\circ} \mathrm{C}$ before use. Plasma was then shipped to Eve Technologies, 
and a 65-plex human cytokine/chemokine multiplex immunoassay was performed. The multiplex immunoassay was performed using a Milliplex assay (Merck Millipore) and analyzed using a Bio-Plex 200 (Bio-Rad). Cytokine and chemokine concentrations were calculated using standard curves for each target molecule.

\section{Statistics}

Enrichment of productive TRBV CDR3s was assessed using the immunoSEQ Analyzer. Only clonotypes with a minimum of 5 template reads were included in the analysis. Significance was determined using a 2-sided binomial test with Benjamini-Hochberg multiple-comparisons correction, where $\alpha=0.01$. To assess the correlation between fold change in clonality and pretherapy clonality, a 2-tailed nonparametric Spearman's correlation was used.

\section{Study approval}

The study was performed according to the principles of the Declaration of Helsinki. It was approved by the QIMR Berghofer Medical Research Institute Human Research Ethics Committee, The Prince Charles Hospital Human Research Ethics Committee, and the Royal Adelaide Hospital Research Ethics Committee, and registered under the Australian New Zealand Clinical Trials Registry (ACTRN12613000981729).

\section{Author contributions}

CS, MAN, KKM, SC, RSF, D Chambers, and RK designed the study. CS, D Corvino, LB, SR, PC, MS, and LLT performed experimental work and analysis of the data. All authors contributed to the writing of the manuscript.

\section{Acknowledgments}

This work was supported by the National Health and Medical Research Council (APP1132519 and APP1062074). CS and RK receive research and consultancy funding from Atara Biotherapeutics Inc.

Address correspondence to: Corey Smith, QIMR Berghofer Medical Research Institute, 300 Herston Road, Herston, Queensland 4029, Australia. Phone: 61.7.3362.0313; Email: corey.smith@ qimrberghofer.edu.au.
1. Crough T, Khanna R. Immunobiology of human cytomegalovirus: from bench to bedside. Clin Microbiol Rev. 2009;22(1):76-98.

2. Cannon MJ, Schmid DS, Hyde TB. Review of cytomegalovirus seroprevalence and demographic characteristics associated with infection. Rev Med Virol. 2010;20(4):202-213.

3. Wang EC, Moss PA, Frodsham P, Lehner PJ, Bell JI, Borysiewicz LK. CD $8^{\text {high }} \mathrm{CD} 57^{+} \mathrm{T}$ lymphocytes in normal, healthy individuals are oligoclonal and respond to human cytomegalovirus. JImmunol.1995;155(10):5046-5056.

4. Bitmansour AD, et al. Clonotypic structure of the human $\mathrm{CD}^{+}{ }^{+}$memory $\mathrm{T}$ cell response to cytomegalovirus. JImmunol. 2001;167(3):1151-1163.

5. Casazza JP, et al. Acquisition of direct antiviral effector functions by CMV-specific $\mathrm{CD} 4^{+} \mathrm{T}$ lymphocytes with cellular maturation. J Exp Med. 2006;203(13):2865-2877.

6. Appay V, et al. Memory CD8 ${ }^{+} \mathrm{T}$ cells vary in differentiation phenotype in different persistent virus infections. Nat Med. 2002;8(4):379-385.

7. Klarenbeek PL, et al. Deep sequencing of antiviral T-cell responses to HCMV and EBV in humans reveals a stable repertoire that is maintained for many years. PLoS Pathog. 2012;8(9):e1002889.

8. Manuel O, et al. Assessment of cytomegalovirus-specific cell-mediated immunity for the prediction of cytomegalovirus disease in high-risk solid-organ transplant recipients: a multicenter cohort study. Clin Infect Dis. 2013;56(6):817-824.

9. Kumar D, et al. Cell-mediated immunity to predict cytomegalovirus disease in high-risk solid organ transplant recipients. Am J Transplant. 2009;9(5):1214-1222.

10. Chiereghin A, et al. Monitoring of cytomegalovirus (CMV)-specific cell-mediated immunity in heart transplant recipients: clinical utility of the QuantiFERON-CMV assay for management of posttransplant CMV infection. J Clin Microbiol. 2018;56(4):e01040-17.
11. Bunde T, et al. Protection from cytomegalovirus after transplantation is correlated with immediate early 1-specific CD8 T cells. J Exp Med. 2005;201(7):1031-1036.

12. Crough T, et al. Symptomatic and asymptomatic viral recrudescence in solid-organ transplant recipients and its relationship with the antigen-specific CD8(+) T-cell response. J Virol. 2007;81(20):11538-11542.

13. Kotton $\mathrm{CN}$, et al. Updated international consensus guidelines on the management of cytomegalovirus in solid-organ transplantation. Transplantation. 2013;96(4):333-360.

14. Eid AJ, Arthurs SK, Deziel PJ, Wilhelm MP, Razonable RR. Emergence of drug-resistant cytomegalovirus in the era of valganciclovir prophylaxis: therapeutic implications and outcomes. Clin Transplant. 2008;22(2):162-170.

15. Holmes-Liew CL, et al. Adoptive T-cell immunotherapy for ganciclovir-resistant CMV disease after lung transplantation. Clin Transl Immunology. 2015;4(3):e35.

16. Pierucci P, Malouf M, Glanville AR, Beagley L, Smith C, Khanna R. Novel autologous T-cell therapy for drug-resistant cytomegalovirus disease after lung transplantation. J Heart Lung Transplant. 2016;35(5):685-687.

17. Smith C, et al. Autologous adoptive T-cell therapy for recurrent or drug-resistant cytomegalovirus complications in solid organ transplant recipients: a single-arm open-label phase I clinical trial. Clin Infect Dis. 2019;68(4):632-640.

18. Glanville J, et al. Identifying specificity groups in the T cell receptor repertoire. Nature. 2017;547(7661):94-98.

19. Smith C, et al. Endogenous antigen presentation impacts on T-box transcription factor expression and functional maturation of $\mathrm{CD}^{+} \mathrm{T}$ cells. Blood. 2012;120(16):3237-3245.

20. Wang EC, Taylor-Wiedeman J, Perera P, Fisher J, Borysiewicz LK. Subsets of CD8 ${ }^{+}, \mathrm{CD}^{+} 7^{+}$cells in normal, healthy individuals: correlations with human cytomegalovirus (HCMV) carrier status, phenotypic and functional analyses. Clin Exp Immunol. 1993;94(2):297-305.

21. Emerson RO, et al. Immunosequencing identifies signatures of cytomegalovirus exposure history and HLA-mediated effects on the T cell repertoire. Nat Genet. 2017;49(5):659-665.

22. Suessmuth $\mathrm{Y}$, et al. CMV reactivation drives posttransplant $\mathrm{T}$-cell reconstitution and results in defects in the underlying TCR $\beta$ repertoire. Blood. 2015;125(25):3835-3850.

23. Wynn KK, et al. Narrowing of T-cell receptor $\beta$ variable repertoire during symptomatic herpesvirus infection in transplant patients. Immunol Cell Biol. 2010;88(2):125-135.

24. Hardy K, et al. Epigenetic programming of T cells impacts immune reconstitution in hematopoietic stem cell transplant recipients. Blood Adv. 2018;2(6):656-668.

25. Gerna G, et al. Monitoring of human cytomegalovirus-specific CD4 and CD8 T-cell immunity in patients receiving solid organ transplantation. Am J Transplant. 2006;6(10):2356-2364.

26. Radha R, et al. Cellular immune responses to cytomegalovirus in renal transplant recipients. Am J Transplant. 2005;5(1):110-117.

27. Walker S, et al. Ex vivo monitoring of human cytomegalovirus-specific CD8 ${ }^{+} \mathrm{T}$-cell responses using QuantiFERON-CMV. Transpl Infect Dis. 2007;9(2):165-170.

28. Gratama JW, et al. Immune monitoring with iTAg MHC Tetramers for prediction of recurrent or persistent cytomegalovirus infection or disease in allogeneic hematopoietic stem cell transplant recipients: a prospective multicenter study. Blood. 2010;116(10):1655-1662.

29. El Haddad L, et al. The ability of a cytomegalovirus ELISPOT assay to predict outcome of low-level CMV reactivation in hematopoietic cell transplant recipients. JInfect Dis. 2019;219(6):898-907.

30. Popescu I, Pipeling MR, Shah PD, Orens JB, 


\section{CLINICAL MEDICINE}

McDyer JF. T-bet:Eomes balance, effector function, and proliferation of cytomegalovirusspecific $\mathrm{CD} 8^{+} \mathrm{T}$ cells during primary infection differentiates the capacity for durable immune control. J Immunol. 2014;193(11):5709-5722.

31. Popescu I, et al. IL-12-dependent cytomegalovirus-specific $\mathrm{CD} 4^{+} \mathrm{T}$ cell proliferation, T-bet induction, and effector multifunction during primary infection are key determinants for early immune control. JImmunol. 2016;196(2):877-890.

32. Rosenberg SA, Dudley ME. Adoptive cell therapy for the treatment of patients with metastatic melanoma. Curr Opin Immunol. 2009;21(2):233-240.

33. Louis $\mathrm{CU}$, et al. Enhancing the in vivo expansion of adoptively transferred EBV-specific CTL with lymphodepleting CD 45 monoclonal antibodies in NPC patients. Blood. 2009;113(11):2442-2450.

34. Miconnet I, et al. Large TCR diversity of virus-specific $\mathrm{CD} 8 \mathrm{~T}$ cells provides the mechanistic basis for massive TCR renewal after antigen exposure. JImmunol. 2011;186(12):7039-7049.

35. Miles JJ, et al. Antigen-driven patterns of TCR bias are shared across diverse outcomes of human hepatitis $\mathrm{C}$ virus infection. J Immunol. 2011;186(2):901-912.

36. Feuchtinger $\mathrm{T}$, et al. Adoptive transfer of pp65-specific $\mathrm{T}$ cells for the treatment of chemorefractory cytomegalovirus disease or reactivation after haploidentical and matched unrelated stem cell transplantation. Blood. 2010;116(20):4360-4367.

37. Koehne $\mathrm{G}$, et al. Immunotherapy with donor $\mathrm{T}$
The Journal of Clinical Investigation

cells sensitized with overlapping pentadecapeptides for treatment of persistent cytomegalovirus infection or viremia. Biol Blood Marrow Transplant. 2015;21(9):1663-1678.

38. Carlson CS, et al. Using synthetic templates to design an unbiased multiplex PCR assay. Nat Commun. 2013;4:2680.

39. Robins HS, et al. Comprehensive assessment of T-cell receptor beta-chain diversity in alphabeta T cells. Blood. 2009;114(19):4099-4107.

40. Shugay M, et al. VDJtools: unifying post-analysis of T cell receptor repertoires. PLoS Comput Biol. 2015;11(11):e1004503.

41. Nazarov VI, et al. tcR: an R package for T cell receptor repertoire advanced data analysis. $B M C$ Bioinformatics. 2015;16:175. 